\title{
RESEARCH
}

Open Access

\section{Tumor size measured by multidetector CT in resectable colon cancer: correlation with regional lymph node metastasis and $\mathrm{N}$ stage}

Anna Mou ${ }^{1,2+}$, Hang Li ${ }^{1,2^{*}}$, Xiao-li Chen ${ }^{3 \dagger}$, Yang-hua Fan ${ }^{4}$ and Hong Pu ${ }^{1,2}$

\begin{abstract}
Background: Lymph node metastasis (LNM) is a risk factor for poor long-term outcomes and a prognostic factor for disease-free survival in colon cancer. Preoperative lymph node status evaluation remains a challenge. The purpose of this study is to determine whether tumor size measured by multidetector computed tomography (MDCT) could be used to predict LNM and N stage in colon cancer.

Material and methods: One hundred six patients with colon cancer who underwent radical surgery within 1 week of MDCT scan were enrolled. Tumor size including tumor length (Tlen), tumor maximum diameter (Tdia), tumor maximum cross-sectional area (Tare), and tumor volume (Tvol) were measured to be correlated with pathologic LNM and N stage using univariate logistic regression analysis, multivariate logistic analysis, and receiver operating characteristic (ROC) curve analysis.

Results: The inter- and intraobserver reproducibility of Tlen (intraclass correlation coefficient [ICC] $=0.94,0.95$, respectively), Tdia (ICC $=0.81,0.93$, respectively), Tare (ICC $=0.97,0.91$, respectively), and Tvol (ICC $=0.99,0.99$, respectively) parameters measurement are excellent. Univariate logistic regression analysis showed that there were significant differences in Tlen, Tdia, Tare, and Tvol between positive and negative LNM $(p<0.001,0.001,<0.001$, $<0.001$, respectively). Multivariate logistic regression analysis revealed that Tvol was independent risk factor for predicting LNM (odds ratio, 1.082; 95\% confidence interval for odds ratio, 1.039, 1.127, $p<0.001$ ). Tlen, Tdia, Tare, and Tvol could distinguish N0 from N1 stage ( $p<0.001,0.041,<0.001,<0.001$, respectively), N0 from N2 (all $p<0.001$ ), No from N1-2 $(p<0.001,0.001,<0.001,<0.001$, respectively), and N0-1 from N2 $(p<0.001,0.001,<0.001,<0.001$, respectively). The area under the ROC curve (AUC) was higher for Tvol than that of Tlen, Tdia, and Tare in identifying LNM ( $A \cup C=0.83,0.82,0.69,0.79$ ), and distinguishing NO from N1 stage (AUC $=0.79,0.78,0.63,0.74$ ), N0 from N2 stage ( $A \cup C=0.92,0.89,0.80,0.89$, respectively), and N0-1 from N2 stage (AUC $=0.84,0.79,0.76,0.83$, respectively).
\end{abstract}

\footnotetext{
*Correspondence: lihang111222@126.com

${ }^{+}$Anna Mou and Xiao-li Chen contributed equally to this work.

'Department of Radiology, Sichuan Academy of Medical Sciences and

Sichuan Provincial People's Hospital, 32\# Second Section of First Ring Rd,

Qingyang District, Chengdu 610072, China

${ }^{2}$ Chinese Academy of Sciences Sichuan Translational Medicine Research

Hospital, Chengdu 610072, China

Full list of author information is available at the end of the article
}

C C The Author(s). 2021 Open Access This article is licensed under a Creative Commons Attribution 4.0 International License, which permits use, sharing, adaptation, distribution and reproduction in any medium or format, as long as you give appropriate credit to the original author(s) and the source, provide a link to the Creative Commons licence, and indicate if changes were made. The images or other third party material in this article are included in the article's Creative Commons licence, unless indicated otherwise in a credit line to the material. If material is not included in the article's Creative Commons licence and your intended use is not permitted by statutory regulation or exceeds the permitted use, you will need to obtain permission directly from the copyright holder. To view a copy of this licence, visit http://creativecommons.org/licenses/by/4.0/ The Creative Commons Public Domain Dedication waiver (http://creativecommons.org/publicdomain/zero/1.0/) applies to the data made available in this article, unless otherwise stated in a credit line to the data. 
Conclusion: Tumor size was correlated with regional LNM in resectable colon cancer. In particularly, Tvol showed the most potential for noninvasive preoperative prediction of regional LNM and N stage.

Keywords: Colon cancer, Lymph node metastasis, Enhanced CT, Tumor size, N stage

\section{Introduction}

Colorectal cancer ranks third, with an estimated 1.8 million new cases, among common cancers worldwide. It is the second most common cause of cancer death, with an estimated 881,000 deaths from colorectal cancer in 2018, accounting for approximately $1 / 10$ of cancer cases and deaths $[1,2]$. Lymph node metastasis (LNM) is nearly always associated with poor long-term outcomes. Patients who have more positive lymph node involvement have lower 5-year survival rates compared to those with less lymph node involvement [3]. The 5-year overall survival rates were $83 \%, 76 \%$, and $54 \%$ for patients with N0, N1, and N2 disease, respectively [4]. Moreover, according to the 7th edition of the American Joint Committee on Cancer (AJCC) staging system and the National Comprehensive Cancer Network (NCCN) Clinical Practice Guidelines for colon cancers, all patients with cT1N0M0 disease can directly receive transanal endoscopic microsurgery, while those with cT2NOM0 or cT3NOMO disease can be recommended to undergo radical resection of colon cancer. Patients with LNM, including those with cT1-3N1M0 disease, are strongly recommended for preoperative chemoradiation therapy $[5,6]$. Accurate noninvasive assessment of LNM and $\mathrm{N}$ stage preoperatively is crucial for effective treatment plans and predicting survival in patients with colon cancer [6].

Endorectal ultrasound and magnetic resonance imaging are widely used in the staging of rectal cancer. However, they are limited in colon cancer because of low sensitivity $[7,8]$. In contrast, computed tomography (CT) is the most common method to preoperatively stage colon cancer because it can describe primary tumor shape, size, location, relationship with surrounding tissues, and the presence of distant metastasis due to its advantages of high tissue resolution, rapid scanning, and convenient follow-up [9-12]. Currently, evaluation of preoperative lymph node status based on morphological features shows poor performance, with a sensitivity of $71 \%$ and specificity of $67 \%$ because lymph node enlargement which might be caused by inflammation and microscopic metastases in small lymph nodes are difficult for radiologists to characterize [12]. The accuracy of CT for detecting LNM is 61-67\% [13]. Lymph node size and shape, as a conventional method, are not reliable indicators for $\operatorname{LNM}[14,15]$. To address these issues, tumor volumetry measured on CT has been studied as a tool for predicting LNM and tumor response to therapy in rectal cancer, esophageal squamous cell carcinoma (ESCC), and gastric carcinoma [16-20]. Therefore, the purpose of this study was to determine whether tumor size measured by multidetector CT could be used to predict regional LNM and $\mathrm{N}$ stage in patients with colon cancer.

\section{Materials and methods}

Ethical approval for this retrospective study was granted by the institutional review board, and the requirement for patient consent was waived because of the retrospective nature of the study.

\section{Patients}

Between December 2016 and June 2018, one hundred and fifty patients diagnosed with colon cancer in our hospital were enrolled in this retrospective analysis (Fig. 1). The inclusion criteria for this study were as follows: (1) immunohistochemical testing and biopsyproven colon cancer; (2) patient did not receive any tumor-related treatment (e.g., radiation therapy or chemotherapy) before CT; (3) tumor was considered resectable and there were no contraindications to surgery; (4) patient was subjected to contrast $\mathrm{CT}$ and visible colon cancer was detected on CT images. Exclusion criteria were as follows: patient did not receive preoperative enhancement $\mathrm{CT}$ scan $(n=20)$; the quality of the CT images was poor, or no tumor was visible on CT images $(n=8)$; preoperative chemotherapy or radiotherapy $(n=16)$. The remaining 106 patients (mean age of 62.93 years old; range from 1883 years old) constituted the study population. According to the 7th AJCC criteria, patients were classified as having stage N0 disease if there were no metastatic lymph nodes. Stage N1a is defined as one metastatic lymph node, N1b includes two to three metastatic lymph nodes, and N1c is defined with a tumor deposited under the serosa, in the mesentery, or in the pericolonic/rectal tissues without peritoneal coverage. N2a is defined by four to six metastatic lymph nodes, and N2b includes more than seven metastatic lymph nodes [5]. Ultimately, there were 52 patients with LNM, defined as positive LNM $(\mathrm{N}+)$; of these 52 patients, 35 patients had N1 disease, including 13 patients with N1a, 15 with N1b, and 7 with N1c. 17 patients had N2 stage, including 11 with N2a and 6 with N2b stage. There were 54 patients without lymph node involvement, which defined as negative lymph node involvement (NO). 


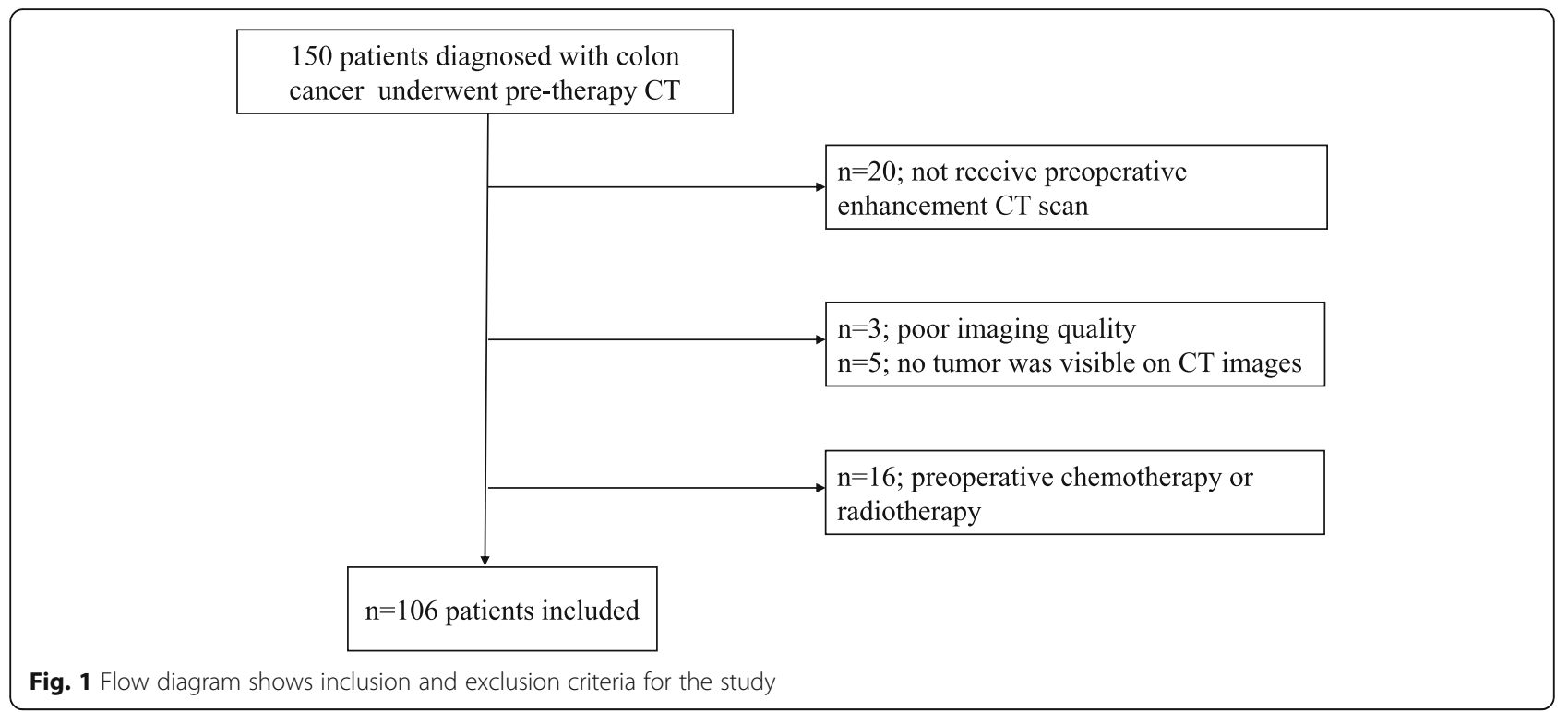

\section{CT technique}

All patients underwent contrast CT on a 64-section multidetector CT system and were asked to adhere to a liquid or semiliquid diet and not to eat after eight o'clock at night the day before. Before CT image acquisition, $1000 \mathrm{~mL}$ of water was given orally every hour to distend the colon and increase the intestinal contrast. In order to minimize the peristaltic bowel movement, all patients received $10 \mathrm{mg}$ of butylscopolamine bromide (Buscopan; Boehringer Ingelheim, Ingelheim, Germany) before the CT examination. Patients were performed in the supine position. The CT scanning variables were 120 $\mathrm{kVp}, 200-380 \mathrm{~mA}$, section thickness of $8 \mathrm{~mm}$, and reconstruction interval of 1-2 $\mathrm{mm}$. CT scanning was performed during the arterial phase (25-30 s) and portal venous phase (60-70 s) after initiation of the contrast material injection (Ultravist 300, Iopamidol; Bayer Healthcare, Berlin, Germany) with a rate of $3 \mathrm{~mL} / \mathrm{s}$, and anatomic coverage was from the thorax to the pelvic cavity covering the entire colon.

\section{Tumor size parameters measurement}

All data were reviewed and measured on Workstation 4.4 (Advantage Workstation version 4.4; General Electric Healthcare). Coronal and sagittal views were reestablished. The tumor margin was delineated by different enhancement of abnormal wall thickening and normal adjacent colon wall on the contrast CT images and corresponding noncontrast CT images. Parameters were measured on 2D images manually, as follows: (1) Tumor length (Tlen): the longest longitudinal diameter of the tumor in coronal or sagittal plane; (2) Tumor maximum diameter (Tdia): the maximum diameter of the tumor in the axial plane which is perpendicular to colonic wall; (3) Tumor maximum cross-sectional area (Tare): regions of interest (ROIs) of tumor area were drawn by tracing the lesion boundary in the axial view; (4) Tumor volume $(\mathrm{Tvol})=$ sum of each axial area of ROIs of tumor $\times$ slice thickness. Pericolic lymph nodes, vessels, adjacent viscera, and lumen were carefully excluded (Fig. 2).

To estimate the accuracy of the measurement in colon cancer, patients were analyzed blindly by an experienced radiologist with 9 years of experience in abdominal radiology (H.L.) and a radiologist with 5 years of experience in radiology measurement (A.M.) for testing interobserver repeatability. The patient measurements were repeated 2 months later by a radiologist (A.M.) in order to verify the intraobserver repeatability.

\section{$\mathrm{CT}$ imaging qualitative analysis}

The same two radiologists also evaluated the regional lymph node status with previous reported criteria [13, 15]. Lymph node positive was defined when the longest lymph node diameter was $>1.0 \mathrm{~cm}$ or $<1.0 \mathrm{~cm}$ in size with round shape, heterogeneity, eccentricity, hilar thinning, calcification, central necrosis, or perinodal infiltration.

\section{Statistical analysis}

All statistical analyses were performed by SPSS software (version 22.0 for Windows; SPSS, Chicago). $P<0.05$ was considered a significant difference. Interobserver and intraobserver measurement agreement were analyzed by calculating the intraclass correlation coefficient (ICC) (0-0.20, poor correlation; $0.21-0.40$, fair correlation; 0.41-0.60, moderate correlation; 0.61-0.80, good 

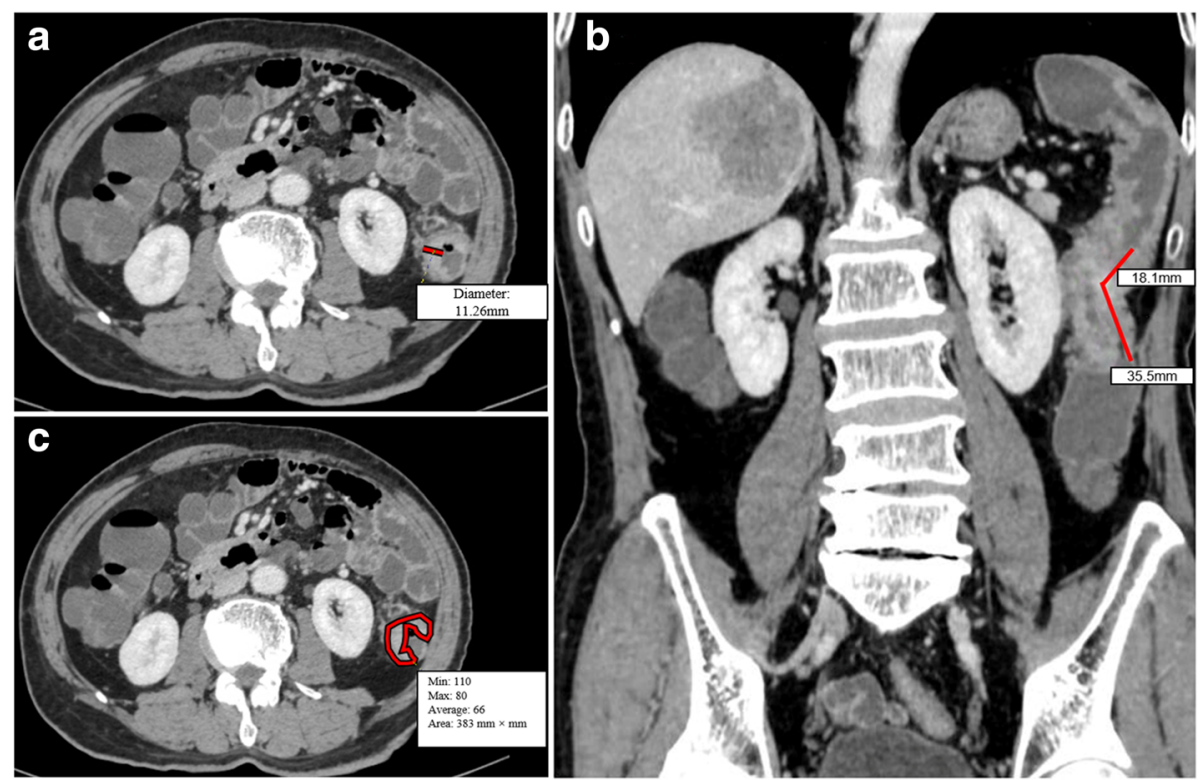

Fig. 2 Tumor size measurement using enhanced CT in a 73-year-old man with colon cancer. a Tumor maximum diameter (Tdia) which is perpendicular to the colonic wall. b The reconstructed coronal image to measure tumor length (Tlen) with a broken line. c The manually drawn area along the margin of the tumor, and the value of this area $\left(383 \mathrm{~mm}^{2}\right)$ is automatically derived by software together with minimal, maximal, and average $\mathrm{CT}$ attenuation (in Hounsfield units)

correlation; and 0.81-1.00, excellent correlation). The relationship between tumor size and LNM were performed by Spearman rank correlation test. Clinicopathologic factors including age, sex, differentiation status, tumor location, vascular carcinoma embolus invasion, neural infiltration, and $\mathrm{T}$ stage were performed by univariate logistic regression analysis. Factors associated with a significant $p$ value were entered in multivariate logistic regression analysis. Preoperative conventional CT-based lymph node status also was tested in multivariate logistic regression analysis. Mann-Whitney $\mathrm{U}$ test and receiver operating curve (ROC) characteristic analysis were performed to determine whether Tlen, Tdia, Tarea, and Tvol could predict LNM and differentiate $\mathrm{N}$ stage. Differences in diagnostic performance were calculated by comparing the AUCs according to the method described by DeLong et al. [21].

\section{Results}

Inter- and intraobserver variability of Tlen, Tdia, Tare, and Tvol measurements

The ICC of interobserver measurement for Tlen, Tdia, Tare, and Tvol is 0.94, 0.81, 0.97, and 0.99, respectively. The ICC of the intraobserver measurement by one observer is $0.95,0.93,0.91$, and 0.99 for Tlen, Tdia, Tare, and Tvol, respectively.

\section{Clinicopathologic factors associated with LNM}

The correlation between clinicopathologic factors with LNM is shown in Table 1. Age, sex, location of colon cancer, vascular carcinoma embolus invasion, and neural infiltration exhibited no significant differences between positive and negative LNM ( $p=0.711,0.123,0.415$, 0.379 , and 0.712 , respectively). There were significant differences in degrees of differentiation, $\mathrm{T}$ stage, Tlen, Tdia, Tare, and Tvol between negative and positive LNM $(p=0.028,0.005,<0.001,0.001,<0.001,<0.001$, respectively) (Table 1). According to multivariate logistic regression analysis, Tvol was independent risk factors for predicting LNM ( $p<0.001$; odds ratio, 1.082; 95\% confidence interval for odds ratio, 1.039, 1.127). In addition, the correlation between $\mathrm{T}$ stage and Tlen, Tdia, Tare, and Tvol is not high and correlation coefficient is less than 0.5 by Spearman test $(r=0.34,0.19,0.37,0.36$ respectively). Therefore, we thought that multicollinearity problems can be ignored when performing the multivariate logistic regression analysis.

\section{Diagnostic performance for Tlen, Tdia, Tare, and Tvol in differentiation of $\mathrm{N}$ stage}

Tlen, Tdia, Tare, and Tvol were correlated with LNM ( $r$ $=0.564,0.369,0.540,0.610$, respectively; all $p<0.001)$. There were significant differences in Tlen, Tdia, Tare, and Tvol between N0 and N1 stage $(p<0.001,0.041$, < $0.001,<0.001$, respectively), N0 and N2 (all $p<0.001$ ), N0 and N1-2 $(p<0.001,0.001,<0.001,<0.001$, respectively), and between N0-1 and N2 ( $p<0.001,0.001$, < $0.001,<0.001$, respectively). AUC, sensitivity, specificity, accuracy values of Tlen, Tlen, Tare, and Tvol in the differentiation of $\mathrm{N}$ stage in colon cancer are 
Table 1 Univariate logistic regression analysis of clinicopathologic factors and tumor size associated with regional lymph node metastasis in colon cancer

\begin{tabular}{|c|c|c|c|}
\hline Parameter & $\begin{array}{l}\text { Positive lymph nodes } \\
(N=52)\end{array}$ & $\begin{array}{l}\text { Negative lymph nodes } \\
(N=54)\end{array}$ & $P$ value \\
\hline Age (years) & $62.46 \pm 11.68$ & $63.39 \pm 12.93$ & 0.711 \\
\hline Sex & & & 0.123 \\
\hline M & $24(46.15 \%)$ & $33(61.11 \%)$ & \\
\hline $\mathrm{F}$ & $28(53.85 \%)$ & $21(38.89 \%)$ & \\
\hline Differentiation & & & 0.028 \\
\hline Well & $2(3.85 \%)$ & $5(9.26 \%)$ & \\
\hline Moderately & $31(59.61 \%)$ & $41(75.93 \%)$ & \\
\hline Poorly & $19(36.54 \%)$ & $8(14.81 \%)$ & \\
\hline Location & & & 0.415 \\
\hline Left colon cancer & $20(38.46 \%)$ & $25(46.30 \%)$ & \\
\hline Right colon cancer & $32(61.54 \%)$ & $29(53.70 \%)$ & \\
\hline Vascular carcinoma embolus invasion & & & 0.379 \\
\hline Positive & $10(19.23 \%)$ & $7(12.96 \%)$ & \\
\hline Negative & $42(80.77 \%)$ & $47(87.04 \%)$ & \\
\hline Neural infiltration & & & 0.712 \\
\hline Positive & $7(6.38 \%)$ & $6(8.51 \%)$ & \\
\hline Negative & $45(93.62 \%)$ & $48(91.49 \%)$ & \\
\hline T staging & & & 0.005 \\
\hline T1 & 0 & $4(7.4 \%)$ & \\
\hline $\mathrm{T} 2$ & $1(1.92 \%)$ & $10(18.52 \%)$ & \\
\hline T3 & $47(90.38 \%)$ & $37(68.52 \%)$ & \\
\hline T4a & $4(7.70 \%)$ & $3(5.56 \%)$ & \\
\hline \multicolumn{4}{|l|}{ Tumor size } \\
\hline Tlen (cm) & $6.42 \pm 2.17$ & $4.25 \pm 1.61$ & $<0.001$ \\
\hline Tdia (cm) & $2.29 \pm 1.09$ & $1.67 \pm 0.76$ & 0.001 \\
\hline Tare $\left(\mathrm{cm}^{2}\right)$ & $15.73 \pm 6.44$ & $8.76 \pm 4.84$ & $<0.001$ \\
\hline Tvol $\left(\mathrm{cm}^{3}\right)$ & $65.70 \pm 56.67$ & $23.96 \pm 16.44$ & $<0.001$ \\
\hline
\end{tabular}

summarized in Table 2 and Fig. 3. From ROC analysis, the area under the ROC curve (AUC) was higher for Tvol than that of Tlen, Tdia, and Tare in identifying LNM (AUC $=0.83,0.82,0.69$, 0.79, respectively), and distinguishing N0 from N1 stage (AUC $=0.79,0.78,0.63,0.74$, respectively), distinguishing N0 from N2 stage (AUC $=0.92,0.89,0.80$, 0.89 , respectively), and distinguishing $\mathrm{N} 0-1$ from $\mathrm{N} 2$ (AUC $=0.84,0.79,0.76,0.83$, respectively).

\section{Preoperative conventional CT-based lymph node status correlation with pathologic results}

Preoperative conventional CT-based lymph node status correlation with pathologic results is summarized in Table 3. According to multivariate logistic regression analysis, preoperative conventional CT-based method was a risk factor for LNM ( $p=0.027$; odds ratio, 3.34; 95\% confidence interval $[\mathrm{CI}]$ for odds ratio, $1.145,10.035)$. The AUC, sensitivity, specificity, and accuracy values of the conventional CT-based method for predicting LNM were 0.726 (95\% CI, 0.628-0.825), 71.2\% (95\% CI, 57.7 81.8\%; 37/52), 74.1\% (95\% CI, 61.0 84.0\%; 40/54), 72.6\% (95\% CI, 63.4 80.3\%; 77/106), respectively. The AUC for Tvol was higher than that of conventional CT-based method for predicting LNM (AUC, 0.830 vs $0.726 ; p=0.045$ ).

\section{Discussion}

In this study, our preliminary results showed that tumor size measured on MDCT could be a tool for the initial prediction of LNM in colon cancer. Tvol was an independent risk factor for predicting LNM. Tlen, Tdia, Tare, and Tvol could distinguish between N0 and N1-2, 
Table 2 ROC analysis of Tlen, Tdia, Tare, and Tvol in the differentiation of $\mathrm{N}$ stage in patients with colon cancer

\begin{tabular}{|c|c|c|c|c|c|c|}
\hline Tumor size & Cutoff & AUC $(95 \% \mathrm{Cl})$ & $P$ value & $\begin{array}{l}\text { Sensitivity } \\
(95 \% \mathrm{Cl})\end{array}$ & $\begin{array}{l}\text { Specificity } \\
(95 \% \mathrm{Cl})\end{array}$ & $\begin{array}{l}\text { Accuracy } \\
(95 \% \mathrm{Cl})\end{array}$ \\
\hline \multicolumn{7}{|l|}{ NO VS N1-2 } \\
\hline Tlen $(\mathrm{cm})$ & 5.20 & $0.82(0.74 \sim 0.90)$ & $<0.001$ & $71.2 \%(57.7 \sim 81.8 \%, 37 / 52)$ & $81.5 \%(69.0 \sim 90.0 \%, 44 / 54)$ & $76.4 \%(67.5 \sim 83.5 \%, 81 / 106)$ \\
\hline Tdia $(\mathrm{cm})$ & 1.56 & $0.69(0.58 \sim 0.79)$ & 0.001 & $71.2 \%(57.7 \sim 81.8 \%, 37 / 52)$ & $51.9 \%(38.9 \sim 64.6 \%, 28 / 54)$ & $61.3 \%(51.8 \sim 70.1 \%, 65 / 106)$ \\
\hline Tare $\left(\mathrm{cm}^{2}\right)$ & 9.47 & $0.79(0.70 \sim 0.87)$ & $<0.001$ & $84.6 \%(72.2 \sim 92.3 \%, 44 / 52)$ & $61.1 \%(47.8 \sim 72.3 \%, 33 / 54)$ & $72.6 \%(63.4 \sim 80.3 \%, 77 / 106)$ \\
\hline Tvol $\left(\mathrm{cm}^{3}\right)$ & 38.00 & $0.83(0.74 \sim 0.90)$ & $<0.001$ & $69.2 \%(55.7 \sim 80.2 \%, 36 / 52)$ & $83.3 \%(71.0 \sim 91.2 \%, 45 / 54)$ & $76.4 \%(67.5 \sim 83.5 \%, 81 / 106)$ \\
\hline \multicolumn{7}{|l|}{ NO VS N1 } \\
\hline Tlen (cm) & 4.25 & $0.78(0.68 \sim 0.88)$ & $<0.001$ & $82.9 \%(66.9 \sim 92.3 \%, 29 / 35)$ & $61.1 \%(47.8 \sim 73.0 \%, 33 / 54)$ & $69.7 \%(59.4 \sim 78.3 \%, 62 / 89)$ \\
\hline Tdia (cm) & 1.49 & $0.63(0.51 \sim 0.75)$ & 0.041 & $74.3 \%(57.8 \sim 86.0 \%, 26 / 35)$ & $48.1 \%(35.4 \sim 61.2 \%, 26 / 54)$ & $58.4 \%(48.0 \sim 68.1 \%, 52 / 89)$ \\
\hline Tare $\left(\mathrm{cm}^{2}\right)$ & 9.47 & $0.74(0.64 \sim 0.84)$ & $<0.001$ & $80.0 \%(63.8 \sim 90.2 \%, 28 / 35)$ & $61.1 \%(47.8 \sim 73.0 \%, 33 / 54)$ & $68.5 \%(58.3 \sim 77.3 \%, 61 / 89)$ \\
\hline Tvol $\left(\mathrm{cm}^{3}\right)$ & 23.22 & $0.79(0.70 \sim 0.88)$ & $<0.001$ & $88.6 \%$ (73.5 96.1\%, 31/35) & $57.4 \%(44.2 \sim 69.7 \%, 31 / 54)$ & $69.7 \%(59.4 \sim 78.3 \%, 62 / 89)$ \\
\hline \multicolumn{7}{|l|}{ NO VS N2 } \\
\hline Tlen (cm) & 5.20 & $0.89(0.82 \sim 0.97)$ & $<0.001$ & $94.1 \%(71.1 \sim 100 \%, 16 / 17)$ & $81.5 \%(69.0 \sim 89.8 \%, 44 / 54)$ & $84.5 \%(74.2 \sim 91.3 \%, 60 / 71)$ \\
\hline Tdia (cm) & 1.99 & $0.80(0.70 \sim 0.91)$ & $<0.001$ & $82.4 \%(58.2 \sim 94.6 \%, 14 / 17)$ & $70.4 \%$ (57.1 80.9\%, 38/54) & $74.6 \%(63.4 \sim 83.4 \%, 53 / 71)$ \\
\hline Tare $\left(\mathrm{cm}^{2}\right)$ & 13.41 & $0.89(0.80 \sim 0.97)$ & $<0.001$ & $82.4 \%(58.2 \sim 94.6 \%, 14 / 17)$ & $83.3 \%$ (71.0 91.2\%, 45/54) & $83.1 \%(72.3 \sim 90.2 \%, 59 / 71)$ \\
\hline Tvol $\left(\mathrm{cm}^{3}\right)$ & 38.40 & $0.92(0.86 \sim 0.99)$ & $<0.001$ & $94.1 \%(71.1 \sim 100 \%, 16 / 17)$ & $83.3 \%$ (71.0 91.2\%, 45/54) & $85.6 \%(75.8 \sim 92.4 \%, 61 / 71)$ \\
\hline \multicolumn{7}{|l|}{ N0-1 VS N2 } \\
\hline Tlen $(\mathrm{cm})$ & 5.20 & $0.79(0.70 \sim 0.88)$ & $<0.001$ & $94.1 \%(71.1 \sim 100 \%, 16 / 17)$ & $65.2 \%(54.8 \sim 74.3 \%, 58 / 89)$ & $69.8 \%(60.5 \sim 77.8 \%, 74 / 106)$ \\
\hline Tdia $(\mathrm{cm})$ & 2.00 & $0.76(0.66 \sim 0.86)$ & 0.001 & $82.4 \%(58.2 \sim 94.6 \%, 14 / 17)$ & $67.4 \%(57.1 \sim 76.3 \%, 60 / 89)$ & $69.8 \%(60.5 \sim 77.8 \%, 74 / 106)$ \\
\hline Tare $\left(\mathrm{cm}^{2}\right)$ & 13.41 & $0.83(0.72 \sim 0.93)$ & $<0.001$ & $82.4 \%(58.2 \sim 94.6 \%, 14 / 17)$ & $75.3 \%(65.3 \sim 85.2 \%, 67 / 89)$ & $76.4 \%(67.5 \sim 83.5 \%, 81 / 106)$ \\
\hline Tvol $\left(\mathrm{cm}^{3}\right)$ & 38.63 & $0.84(0.76 \sim 0.93)$ & $<0.001$ & $94.1 \%(71.1 \sim 100 \%, 16 / 17)$ & $68.5 \%(58.3 \sim 77.3 \%, 61 / 89)$ & $72.6 \%(63.4 \sim 80.3 \%, 77 / 106)$ \\
\hline
\end{tabular}

Note: Tdia tumor maximum diameter, Tlen tumor length, Tare tumor maximum cross-sectional area, Tvol tumor volume, AUC the area under the ROC curve, $\mathrm{Cl}$ confidence interval

N0 and N1, N0 and N2, and N0-1 and N2 stage. Tvol had the best diagnostic efficiency in identifying LNM and differentiating $\mathrm{N}$ stage.

Previous studies have applied different criteria based on either size and/or morphology for identifying LNM [22-24]. Another study used the radiographic criteria of lymph node diameter $(>1.0 \mathrm{~cm})$ or round shape, heterogeneity, eccentricity, hilar thinning, calcification, central necrosis, and perinodal infiltration to evaluate lymph node involvement with sensitivity of $54-88 \%$, specificity of $55-66 \%$, accuracy of $61-70 \%$ [13]. Sibileau et al. reported the accuracy of the association of the 3 criteria (size, number, and density) with sensitivity of $77.3 \%$ and specificity of $77.4 \%$ [25]. However, metastatic lymph node smaller than $3 \mathrm{~mm}$ are difficult to depict with MR imaging and CT imaging and can easily be misinterpreted as small blood vessels and, most important, that some of nodes larger than $1 \mathrm{~cm}$ seen on CT images may be the benign. Therefore, the node seen on CT images that could not be matched with histopathologic findings were all judged to be benign on CT images. This limitation made morphological method not accurate to assess lymph node status. It is hard to ensure the metastatic lymph node we diagnosed on CT images is the one confirmed by pathology eventually.
Tumor length is an independent predictor of mortality in patients with esophageal carcinoma and a risk factor for LNM to predict survival in patients with ESCC [26, 27]. In this study, we indicated that tumor length was correlated with LNM in patients with colon cancer. This was because increasing longitudinal growth in the lymphatic-rich submucosa was a predictor of regional LNM. The longer the tumor length, the deeper the tumor invading the colonic wall and the more frequent the incidence of LNM. Tumor thickness was an independent adverse factor for LNM in oral carcinoma [28] and could be associated with LNM in ESCC [26]. In theory, the larger the tumor diameter, the deeper tumor invading the colon wall, more likely invading adjacent structures, and the higher risk of LNM. In our study, we found that Tdia was correlated with LNM, but the diagnostic efficiency of Tdia is not high in colon cancer. We thought that the inflatable dilatation of the colon and blur margin caused by tumor inflammation and invasion could affect tumor diameter measurement. For Tare, we found that Tare was also correlated with LNM. Tare is the maximum cross-sectional area of colon cancer and is used for delineating the whole circumference of the tumor. Compared with Tdia, it contains a much broader margin and could reflect tumor geometry and growth. 

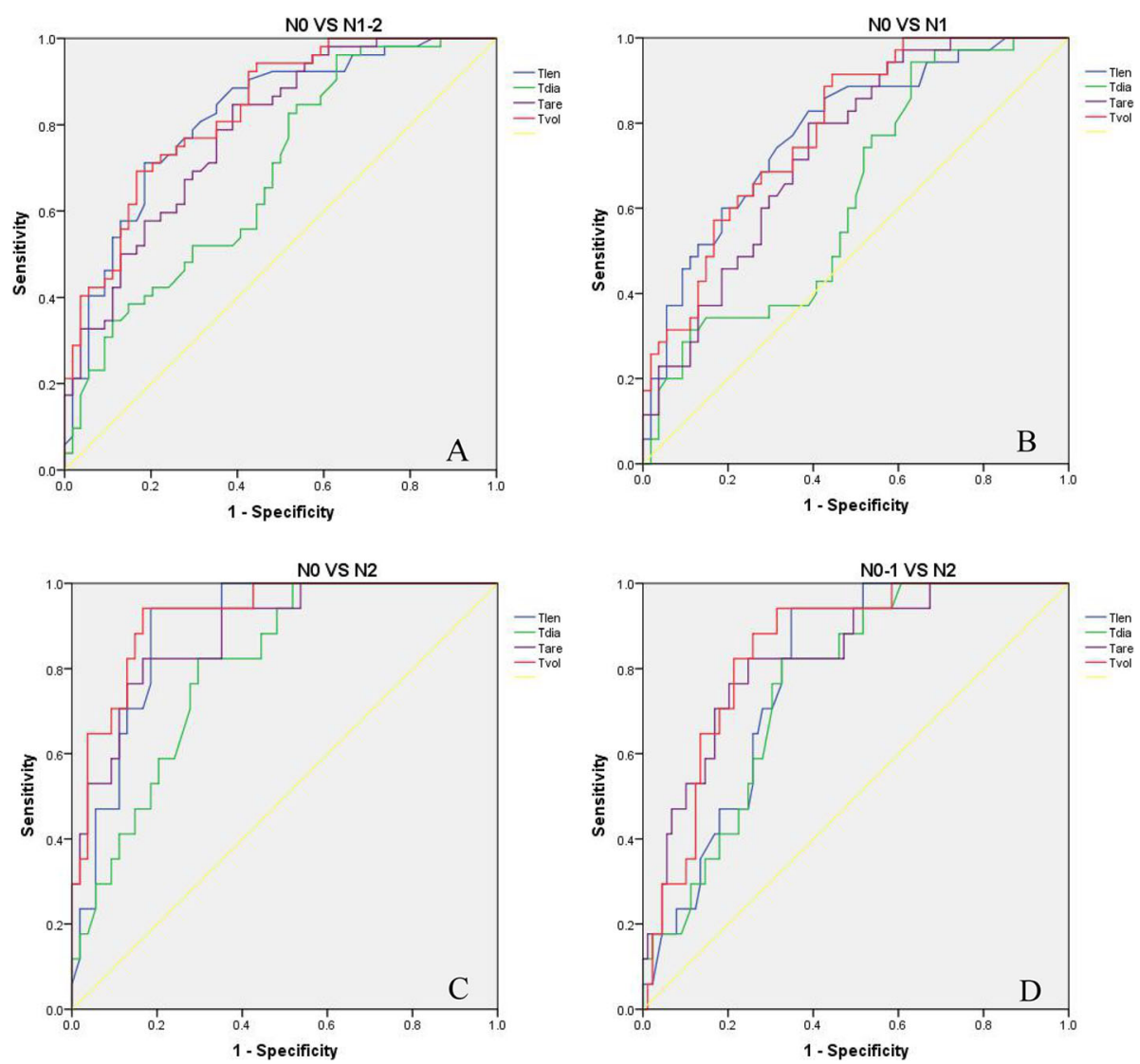

Fig. 3 Receiver-operating curve analysis of tumor length (Tlen), tumor maximum diameter (Tdia), tumor maximum cross-sectional area (Tare), and tumor volume (Tvol) distinguishing N0 from N1-2 (A), N0 from N1 (B), N0 from N2 (C), N0-1 form N2 stage (D) in patients with colon cancer

Previous study reported the sensitivity, specificity, and accuracy of CT colonography for predicting LNM were $69.31 \%, 66.15 \%$, and $67.14 \%$ respectively [29]. In this study, we found that tumor volume had the best repeatability measurement and was an independent risk factor for predicting LNM in patients with colon cancer by multivariate logistic analysis. The sensitivity, specificity, and accuracy of Tvol for predicting LNM were 69.2\%, $83.3 \%$, and $76.4 \%$, respectively. However, some other study indicated tumor CT colonography volumetry could not predict $\mathrm{N}$ stage [30]. The explanation for the different results could be the relatively small sample size of LNM. First, the sample size of patients with LNM was smaller than that of our studies. Second, the sample size of patients with LNM was smaller than the sample size

Table 3 Preoperative CT-based lymph nodes status

\begin{tabular}{llll}
\hline $\begin{array}{l}\text { Preoperative } \\
\begin{array}{l}\text { CT lymph } \\
\text { node status }\end{array}\end{array}$ & Pathologic results & Total \\
\cline { 2 - 3 } & Positive $(\boldsymbol{n}=\mathbf{5 2})$ & Negative $(\boldsymbol{n}=\mathbf{5 4})$ & \\
\hline Positive & 37 & 14 & 51 \\
Negative & 15 & 40 & 55 \\
Total & 52 & 54 & 106 \\
\hline
\end{tabular}

of patients without LNM in the published study. Usually, to be a risk factor is not equal to can be used to predict LNM. The explanation for this reason could be that most of these published studies utilized clinicopathologic factors excluding tumor size to predict LNM and concluded a risk factor is not equal to can be used to predict LNM. Quantitative parameter such as tumor volume had not been included for predicting LNM. Moreover, we demonstrated that Tvol could help differentiate N0 from N1-2, N0 from N1, N0 from N2, and N0-1 from N2 with moderately sensitivity, specificity, and accuracy. Our findings were consistent with these previous studies in other tumors. For example, $\mathrm{Li}$ et al. showed that tumor volume could predict regional LNM and differentiate various $\mathrm{N}$ stages with an accuracy of $70 \%$ in adenocarcinoma of the esophagogastric junction [20]. Tumor volume measured on MDCT could differentiate NO and N1-3 stages, N0-1 and N2-3, and N0-2 and N3 with moderately accurate in resectable ESCC [26]. Chen et al. reported that tumor volume data had better correlation with LNM than that of the tumor length and tumor diameters [31]. We also demonstrated that Tvol had the highest AUC compared with Tlen, Tdia, and Tare in 
distinguishing $\mathrm{N}$ stage. This may be because tumor volume takes both the Tlen, Tdia, and Tare into consideration. The larger the tumor volume, the deeper the tumor invading the colonic wall and the more frequent the incidence of LNM. In addition, treatment of colon cancers in both N1 and N2 category is different. Patients with $\mathrm{N} 2$ category regardless of $\mathrm{T}$ stage should be classified into high-risk stage III. These patients with N2 colon cancer may have a poorer prognosis than those with N1 category [6]. Therefore, patients with N2 colon cancer should undergo more longer adjuvant therapy to reduce recurrence. Although our results indicated tumor size could not be used to distinguish N1 from N2 category, Tvol had good diagnostic efficacy for differentiating N0-1 from N2 with sensitivity of 94.1\%, specificity of $68.5 \%$, and accuracy of $72.6 \%$. In our study, the AUC, sensitivity, specificity, and accuracy values of the conventional CT-based method for distinguishing N0 from N1$\mathrm{N} 2$ were $0.726,71.2 \%, 74.1 \%$, and $72.6 \%$, respectively. Tvol had a better diagnostic efficiency to evaluate LNM, with AUC, sensitivity, specificity, accuracy of 0.83 , $69.2 \%, 83.3 \%$, and $76.4 \%$, respectively. The AUC for Tvol was higher than that of conventional CT-based method for predicting LNM (AUC, 0.830 vs $0.726 ; p=0.045$ ). We can conclude that Tvol may be the best predictor to differentiate $\mathrm{N}$ stage in colon cancer.

Our study had several limitations. First, the collected sample number is too small and only 52 patients had LNM. Small data analysis could lead that the current result do not reflect the whole information. Lymphovascular invasion and perineural invasion have a high risk of LNM and a poor prognostic factor in colon diseases $[6,32,33]$. In our study, just few cases had positive lymphovascular or perineural invasion, which resulted in no significant differences. Second, tumor volume measurements on CT images can be time-consuming. However, in this study, the time required for well-trained radiologists to draw the whole tumor area was controlled to $10 \mathrm{~min}$. In future study, semiautomatic measurement method may be used to reduce the time of measurement. Third, the measurement of Tlen, Tdia, and Tare might be affected by the distention of the contents in the intestinal canal. To minimize this effect, all patients were asked to perform bowel preparation with butylscopolamine bromide and drink $1000 \mathrm{~mL}$ of water before CT scanning.

In conclusion, the manual measurement of tumor length, tumor diameter, tumor area, and tumor volume on MDCT in patients with colon cancer provided good maneuverability and repeatability. Tvol had higher diagnostic efficiency in identifying LNM and differentiating $\mathrm{N} 0$ from N1, N0-1 from N2, and N0 from N2. Tvol could be helpful in quantitatively predicting LNM and $\mathrm{N}$ stage for the appropriate choice of treatment approach for this tumor.

\section{Abbreviations}

MDCT: Multidetector computed tomography; LNM: Lymph node metastasis; Tlen: Tumor length; Tdia: Tumor maximum diameter; Tare: Tumor maximum cross-sectional area; Tvol: Tumor volume; ROC: Receiver operating

characteristic; AUC: Area under the ROC curve; ICC: Intraclass correlation coefficient; ESCC: Esophageal squamous cell carcinoma; AJCC: American Joint Committee on Cancer; NCCN: National Comprehensive Cancer Network;

$\mathrm{Cl}$ : Confidence interval

\section{Acknowledgements \\ None}

\section{Authors' contributions}

$\mathrm{AM}, \mathrm{HL}$, and $\mathrm{XC}$ designed the overall study. AM and HL collected, analyzed the data, and edited the manuscript. YF, HP, and XC provide some advice to supervise this manuscript. The authors read and approved the final manuscript.

\section{Funding}

None

Availability of data and materials

Not applicable.

\section{Declarations}

Ethics approval and consent to participate

The study was approved by the institutional review board of Sichuan Provincial People's Hospital, and the requirement for patient consent was waived because of the retrospective nature of the study.

Consent for publication

Not applicable.

\section{Competing interests}

There is no conflict of interest among all authors.

\section{Author details}

${ }^{1}$ Department of Radiology, Sichuan Academy of Medical Sciences and Sichuan Provincial People's Hospital, 32\# Second Section of First Ring Rd, Qingyang District, Chengdu 610072, China. ${ }^{2}$ Chinese Academy of Sciences Sichuan Translational Medicine Research Hospital, Chengdu 610072, China. ${ }^{3}$ Department of Radiology, Sichuan Cancer Hospital, Chengdu 610072, China. ${ }^{4}$ Department of Neurosurgery, Peking Union Medical College Hospital, Chinese Academy of Medical Sciences and Peking Union Medical College, Beijing 100032, China.

Received: 27 March 2021 Accepted: 4 June 2021

Published online: 16 June 2021

\section{References}

1. Ferlay J, Colombet M, Soerjomataram I, Mathers C, Parkin DM, Piñeros M, et al. Estimating the global cancer incidence and mortality in 2018: GLOBOCAN sources and methods. Int J Cancer. 2019;144(8):1941-53. https://doi.org/10.1002/ijc.31937.

2. Bray F, Ferlay J, Soerjomataram I, Siegel RL, Torre LA, Jemal A. Global cancer statistics 2018: GLOBOCAN estimates of incidence and mortality worldwide for 36 cancers in 185 countries. CA Cancer J Clin. 2018;68(6):394-424. https://doi.org/10.3322/caac.21492.

3. Kuo YT, Tsai WS, Hung HY, Hsieh PS, Chiang SF, Lai CC. Prognostic value of regional lymph node involvement in patients with metastatic colorectal cancer: palliative versus curative resection. World J Surg Oncol. 2021;19(1): 150. https://doi.org/10.1186/s12957-021-02260-Z.

4. Huh JW, Jeong YY, Kim HR, Kim YJ. Prognostic value of preoperative radiological staging assessed by computed tomography in patients with nonmetastatic colon cancer. Ann Oncol. 2012;23(5):1198-206. https://doi. org/10.1093/annonc/mdr404.

5. Edge SB, Byrd DR, Compton CC, Fritz AG, Greene FL, Trotti A. AJCC cancer staging manual (7th ed). New York, NY: Springer; 2010. p. 103-15.

6. Benson $A B$, Venook AP, Al-Hawary MM, Cederquist $L$, Chen YJ, Ciombor KK, et al. Rectal cancer, version 2.2018, NCCN clinical practice guidelines in 
oncology. J Natl Compr Canc Netw. 2018;16(7):874-901. https://doi.org/10. 6004/jnccn.2018.0061.

7. Hofheinz RD, Wenz F, Post S, Matzdorff A, Laechelt S, Hartmann JT, et al. Chemoradiotherapy with capecitabine versus fluorouracil for locally advanced rectal cancer: a randomised, multicentre, noninferiority, phase 3 trial. Lancet Oncol. 2012;13(6):579-88. https://doi. org/10.1016/S1470-2045(12)70116-X.

8. Hötker AM, Tarlinton L, Mazaheri Y, Woo KM, Gönen M, Saltz LB, et al. Multiparametric MRI in the assessment of response of rectal cancer to neoadjuvant chemoradiotherapy: a comparison of morphological, volumetric and functional MRI parameters. Eur Radiol. 2016;26(12):4303-12. https://doi.org/10.1007/s00330-016-4283-9.

9. Bai RJ, Ren SH, Jiang HJ, Li JP, Liu XC, Xue LM. Accuracy of multi-slice spiral computed tomography for preoperative tumor node metastasis (TNM) staging of colorectal carcinoma. Med Sci Monit. 2017;23:3470-9. https://doi. org/10.12659/MSM.902649.

10. Malmstrøm ML, Brisling S, Klausen TW, Săftoiu A, Perner T, Vilmann P, et al. Staging with computed tomography of patients with colon cancer. Int J Colorectal Dis. 2018;33(1):9-17. https://doi.org/10.1007/s00384-017-2932-3.

11. Wang ZH, Ye Y, Hu YT, Han SG, Sun LF, Xu D, et al. Extent of enhancement on multiphase contrast-enhanced $C T$ images is a potential prognostic factor of stage I-III colon cancer. Eur Radiol. 2019;29(3):1114-23. https://doi.org/1 0.1007/s00330-018-5689-3.

12. Nerad E, Lahaye MJ, Maas M, Nelemans P, Bakers FCH, Beets FL, et al. Diagnostic accuracy of $C T$ for local staging of colon cancer: a systematic review and meta-analysis. AJR Am J Roentgenol. 2016;207(5):984-95. https:// doi.org/10.2214/AJR.15.15785.

13. Choi AH, Nelson RA, Schoellhammer HF, Cho W, Ko M, Arrington A, et al. Accuracy of computed tomography in nodal staging of colon cancer patients. World J Gastrointest Surg. 2015;7(7):116-22. https://doi.org/10.424 0/wjgs.v7.i7.116.

14. Mönig SP, Schröder W, Baldus SE, Hölscher AH. Preoperative lymph-node staging in gastrointestinal cancer--correlation between size and tumor stage. Onkologie. 2002;25(4):342-4. https://doi.org/10.1159/000066051.

15. Erik R, Mirna AN, Torbjörn H, Lennart B. Assessment and diagnostic accuracy of lymph node status to predict stage III colon cancer using computed tomography. Cancer imaging. 2017;17:3.

16. Planz VB, Lubner MG, Pickhardt PJ. Volumetric analysis at abdominal CT: oncologic and non-oncologic applications. Br J Radiol. 2019;92(1095): 20180631. https://doi.org/10.1259/bjr.20180631.

17. Chen M, Li X, Chen Y, Liu PP, Chen ZW, Shen MM, et al. Proposed revision of the 8th edition AJCC clinical staging system for esophageal squamous cell cancer treated with definitive chemo-IMRT based on CT imaging. Radiat Oncol. 2019;14(1):54. https://doi.org/10.1186/s13014-019-1258-4.

18. Miyamoto H, Kunisaki C, Sato S, Tanaka Y, Sato K, Kosaka T, et al. Tumor volume index as a prognostic factor in patients after curative esophageal cancer resection. Ann Surg Onco. 2019;26(6):1909-15. https://doi.org/10.124 5/s10434-019-07308-9.

19. Hallinan JTPD, Venkatesh SK, Peter L, Makmur A, Yong WP, So JBY. CT volumetry for gastric carcinoma: association with TNM stage. Eur Radiol. 2014;24(12):3105-14. https://doi.org/10.1007/s00330-014-3316-5.

20. Li R, Chen TW, Hu J, Guo DD, Zhang XM, Deng D, et al. Tumor volume of resectable adenocarcinoma of the esophagogastric junction at multidetector $\mathrm{CT}$ : association with regional lymph node metastasis and $\mathrm{N}$ stage. Radiology. 2013;269(1):130-8. https://doi.org/10.1148/radiol.13122269.

21. DeLong ER, DeLong DM, Clarke-Pearson DL. Comparing the areas under two or more correlated receiver operating characteristic curves: a nonparametric approach. Biometrics. 1988;44(3):837-45. https://doi.org/1 $0.2307 / 2531595$.

22. Rollvén E, Blomqvist L, Öistämö E, Hjern F, Csanaky G, Abraham NM. Morphological predictors for lymph node metastases on computed tomography in colon cancer. Abdom Radiol (NY). 2019;44(5):1712-21. https://doi.org/10.1007/s00261-019-01900-Z.

23. Kumamoto T, Shindoh J, Mita H, Fujii Y, Mihara Y, Takahashi M, et al. Optimal diagnostic method using multidetectorrow computed tomography for predicting lymph node metastasis in colorectal cancer. World J Surg Oncol. 2019;17(1):39. https://doi.org/10.1186/s12957-019-1583-y.

24. Lee J, Park HM, Lee SY, Kim CH, Kim HR. Prognostic significance of enlarged paraaortic lymph nodes detected during left-sided colorectal cancer surgery: a single-center retrospective cohort study. World J Surg Oncol. 2021;19(1):9. https://doi.org/10.1186/s12957-020-02118-w.
25. Sibileau E, Ridereau ZC, Vanel D, Pavageau AH, Bertrais S, Cesbron EM, et al. Accuracy of water-enema multidetector computed tomography (WE-MDCT) in colon cancer staging: a prospective study. Abdom Imaging. 2014;39(5): 941-8. https://doi.org/10.1007/s00261-014-0150-9.

26. Li H, Chen TW, Li ZL, Zhang XM, Chen XL, Wang LY, et al. Tumour size of resectable oesophageal squamous cell carcinoma measured with multidetector computed tomography for predicting regional lymph node metastasis and N stage. Eur Radiol. 2012;22(11):2487-93. https://doi.org/10.1 007/s00330-012-2512-4.

27. Eloubeidi MA, Desmond R, Arguedas MR, Reed CE, Wilcox CM. Prognostic factors for the survival of patients with esophageal carcinoma in the U.S.: the importance of tumor length and lymph node status. Cancer. 2002;95(7): 1434-43. https://doi.org/10.1002/cncr.10868.

28. Pinto FR, de Matos LL, Palermo FC, Kulcsar MAV, Cavalheiro BG, de Mello ES, et al. Tumor thickness as an independent risk factor of early recurrence in oral cavity squamous cell carcinoma. Eur Arch Otorhinolaryngol. 2014;271(6): 1747-54. https://doi.org/10.1007/s00405-013-2704-9.

29. Zhou YD, Han ZW, Dou FF, Yan T. Pre-colectomy location and TNM staging of colon cancer by the computed tomography colonography: a diagnostic performance study. World J Surg Oncol. 2021;19(1):120. https://doi.org/10.11 86/s12957-021-02215-4.

30. Park JY, Kim SH, Lee SM, Lee JS, Han JK. CT volumetric measurement of colorectal cancer helps predict tumor staging and prognosis. PLoS One. 2017;12(6):e0178522. https://doi.org/10.1371/journal.pone.0178522.

31. Chen XL, Chen GW, Pu H, Yin LL, Li ZL, Song B, et al. DWI and T2-weighted $\mathrm{MRI}$ volumetry in resectable rectal cancer: correlation with lymphovascular invasion and lymph node metastases. AJR Am J Roentgenol. 2019;1:1-8.

32. Yang $Y$, Huang $X$, Sun J, Gao $P$, Song $Y$, Chen $X$, et al. Prognostic value of perineural invasion in colorectal cancer: a meta-analysis. J Gastrointest Surg. 2015;19(6):1113-22. https://doi.org/10.1007/s11605-015-2761-z.

33. Gao ZY, Cao HH, Xu X, Wang Q, Wu YG, Lu QC. Prognostic value of lymphovascular invasion in stage II colorectal cancer patients with an inadequate examination of lymph nodes. World J Surg Oncol. 2021: 19(1):125. https://doi.org/10.1186/s12957-021-02224-3.

\section{Publisher's Note}

Springer Nature remains neutral with regard to jurisdictional claims in published maps and institutional affiliations.

Ready to submit your research? Choose BMC and benefit from:

- fast, convenient online submission

- thorough peer review by experienced researchers in your field

- rapid publication on acceptance

- support for research data, including large and complex data types

- gold Open Access which fosters wider collaboration and increased citations

- maximum visibility for your research: over $100 \mathrm{M}$ website views per year

At $\mathrm{BMC}$, research is always in progress.

Learn more biomedcentral.com/submissions 